\title{
Home blood pressure variability: a new target to monitor in chronic kidney disease patients with low eGFR?
}

\author{
Maritza J Romero \\ Hypertension Research (2013) 36, 673-675; doi:10.1038/hr.2013.53; published online 23 May 2013
}

$\mathrm{B}$ lood pressure variability (BPV) in hyperO tensive patients is increasingly attracting the attention of clinicians and scientists as a way to identify patients at a higher risk of cardiovascular events and stroke, and to monitor therapeutic outcomes. However, a disadvantage of using BPV in the routine clinical management of hypertension is the multiple visits that are required. Therefore, monitoring BPV at home before the patient's visit to the clinic is an option to provide physicians with accessibility to BPV measurements to be incorporated into hypertension management.

Variability in daily blood pressure measurements at home has been found to correlate with visit-to-visit variability. ${ }^{1}$ In addition, patients in clinical studies are judged to be compliant. In view of these two premises, the contribution of Nishimura et $a .^{2}$ to this issue is highly relevant.

The results from the study by Nishimura et $a .^{2}$ are based on baseline data from the home blood pressure-diabetic nephropathy (HBP-DN) study. This is a 3-year prospective cohort study that was designed to determine the optimal HBP to improve the prognosis of type 2 diabetic patients with microalbuminuria; the study is supported by a Japanese National Hospital Organization collaborative clinical research grant.

Although it is observational and does not provide specific recommendations with regard to hypertension management for this cohort, the study by Nishimura et $a .^{2}$

MJ Romero is at Department of Pharmacology and Toxicology, Georgia Regents University, Augusta, GA, USA and at the Department of Anesthesiology, Georgia Regents University, Augusta, GA, USA

E-mail: MROMEROLUCAS@gru.edu analyzed baseline data from the HBP-DN study to determine whether a difference exists in the home BPV (HBPV, calculated as the s.d. of morning and evening HBP measurements for 7 days) between two groups of patients with chronic kidney disease (CKD): preserved versus lowestimated glomerular filtration rate (eGFR) groups. Patients with CKD in stages 1 and 2 (eGFR $\geqslant 90$ and $60-89 \mathrm{ml} \mathrm{min}^{-1}$ per $1.73 \mathrm{~m}^{2}$, respectively) were classified as the preserved eGFR group, whereas patients with CKD in stages 3 and 4 (eGFR 30-59 and $15-29 \mathrm{ml} \mathrm{min}^{-1}$ per $1.73 \mathrm{~m}^{2}$, respectively) were classified as the low-eGFR group.

The researchers demonstrated that low eGFR was the explanatory variable for the home BPV, independent of the albumin-tocreatinine ratio, age, gender, duration of diabetes and HBP level. ${ }^{2}$

A previous study ${ }^{3}$ showed that several factors associated with organ damage (albuminuria, eGFR and pulse pressure) also influence visit-to-visit BPV. The study by Nishimura et al. ${ }^{2}$ clearly provides preliminary data that specifically link elevated home systolic blood pressure (HSBP) variability, with low eGFR in type 2 diabetic patients with microalbuminuria. Moreover, the results demonstrate that this association is significantly correlated with a higher incidence of coronary heart disease and retinopathy.

The 2012 update of the Kidney Disease Outcomes Quality Initiative Clinical Practice Guideline for Diabetes and Chronic Kidney Disease ${ }^{4}$ states that strict blood pressure control and ACE inhibitors are more effective in slowing kidney-disease progression in patients with higher baseline proteinuria, with a recommended target blood pressure of $<130 / 80 \mathrm{~mm} \mathrm{Hg}$. On the other hand, the incidence of stroke increases in patients with CKD stages 3 and 4, if SBP decreases below $120 \mathrm{~mm} \mathrm{Hg.}^{5}$

The study by Nishimura et al. ${ }^{2}$ found that the mean of the morning and evening HSBP measurements (the so-called 'all HSBP') was above the recommended target BP goal for CKD patients; the SBP was higher in the morning than in the evening in both the groups. However, no significant differences in these parameters were observed between the low eGFR and the preserved eGFR groups. The same holds true for the morningevening differences in HBP. Therefore, the elevated HSBP variability in the low-eGFR group detected in this study might be derived from factors other than the morning-evening differences in HSBP.

Nishimura et al. ${ }^{2}$ also found that the morning home diastolic blood pressure (HDBP), evening HDBP and all HDBP (mean of the morning and evening HDBP) reached the target DBP in both groups. However, there were no significant differences in the variability of HDBP between the low- and preserved-eGFR groups.

All of these observations strengthen the relevance of the contribution by Nishimura et $a .^{2}$ in addressing the importance of evaluating HBP variability in this cohort with low eGFR, which had a higher incidence of coronary heart disease and retinopathy. A larger cohort study is necessary to determine whether significantly elevated HDBP variability is also present in the low-eGFR group, compared with the preserved eGFR group, as the s.d. of the 
HDBP and the delta HDBP (maximum HDBP - minimum HDBP) were slightly elevated in the low-eGFR group.

The questions that arise from this study are as follows: (i) what would be the target goal to reduce BPV for type 2 diabetes patients with low eGFR and microalbuminuria? (ii) What are the suitable therapies currently available to efficiently control BP at the target goal, in addition to target BPV, for this patient population? (Figure 1).

Nishimura et $a .^{2}$ observed an increased amount of prescribed antihypertensive medications, including more renin-angiotensin system blockade drugs, in the low-eGFR group with higher BPV than in the preserved eGFR group, indicating that conventional or current combinational therapies have poor efficacy in controlling BPV in this cohort.

Antihypertensive agents that have beneficial effects on the progression of CKD or reduce the risk of cardiovascular disease, in addition to their antihypertensive effects, are defined as 'preferred agents' for these conditions. ${ }^{4}$ However, patients with well-controlled blood pressure, but high residual variability in SBP, had a five-time higher risk of stroke than those with low residual SBP variability, as observed in the ASCOT-BPLA trial. ${ }^{6}$ This study also suggests that calcium channel blockers and, to a lesser extent, thiazide diuretics are better drugs for controlling BPV, and preventing stroke and other vascular events.

BPV stabilization is a potentially important target for drug development and combination therapy. As such, novel drugs or combinations of drugs that reduce variability even more effectively than calcium channel blockers could greatly reduce the occurrence of stroke and other cardiovascular complications. Therefore, the challenge for the pharmaceutical industry is to develop flexible dose combinations within one tablet. This will allow physicians to prescribe medications and modify the dosage of the component parts in a single tablet, according to the blood pressure and BPV responses. ${ }^{7}$

Despite the scientific reports showing the strong association and prevalence of stroke in hypertensive patients with high BPV, the observational study performed by Nishimura et $a .^{2}$ found no significant difference in the prevalence of stroke between the preservedand low-eGFR groups, the latter of which presented with higher BPV despite an increased amount of prescribed antihypertensive medications. This could have resulted from the small patient population evaluated in this study, which precludes definitive conclusions but prompts other reasonable questions: (i) are there other variables than high BPV, modifying the prevalence of stroke in hypertensive type 2 diabetic patients? (ii) Would including an evaluation of different uremic toxins be of additional significance when determining the risk or prevalence of stroke in type 2 diabetic patients with microalbuminuria and low eGFR?

A previous study has shown that eGFR is not representative of the concentration of several uremic toxins related to cardiovascular morbidity and mortality. ${ }^{8}$ In addition, BPV has been shown to depend on sympathetic nervous systemmediated behavioral influences on the heart and peripheral circulation, ${ }^{9}$ and a higher sympathetic response has been observed in uremic patients. ${ }^{10}$ Whether these factors are important determinants of differences in the prevalence of stroke in hypertensive patients with or without type 2 diabetes, microalbuminuria and low eGFR has yet to be determined.

Nishimura et al.'s study ${ }^{2}$ suggests a need to perform larger randomized control trials aimed to elucidate definite outcomes in blood pressure control in type 2 diabetes patients with CKD and microalbuminuria, including trials with drugs that target BPV, lower blood pressure and reduce the sympathetic response.

The results of Nishimura et al.'s study ${ }^{2}$ alerted physicians to exercise greater care when treating hypertensive patients with low eGFR and microalbuminuria, so that high-risk patients and those with more variation in day-to-day HBP could be identified. These observations should be confirmed by using follow-up data from the HBP-DN study and from parallel investigations in other patient cohorts.

\section{CONFLICT OF INTEREST}

The author declares no conflict of interest.

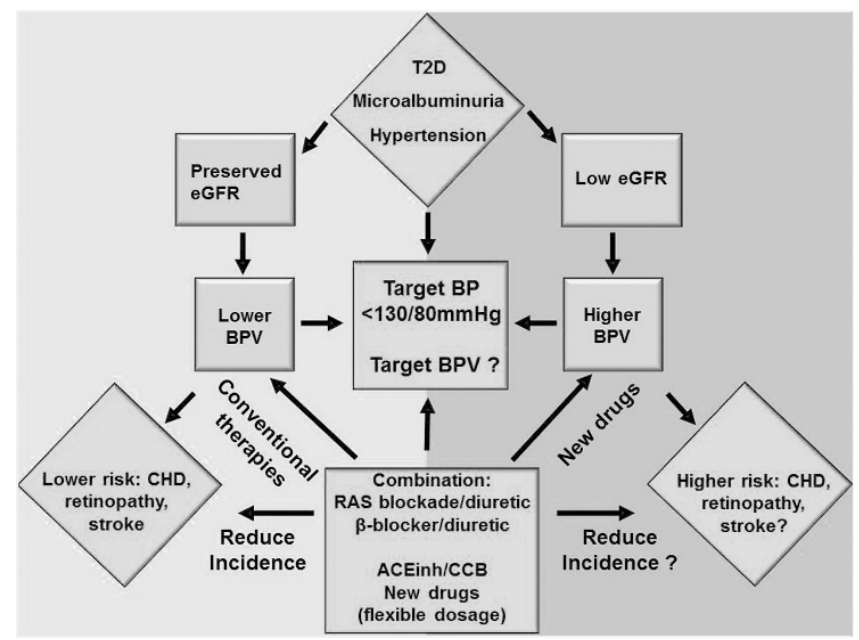

Figure 1 Illustration of BPV as a new target to reduce the incidence of vascular complications in T2D patients with microalbuminuria and low eGFR. Left panel (yellow) represents patients with preserved eGFR who may benefit from conventional therapies. Right panel (pink) represents patients with low eGFR at a higher risk of having high BPV and with an increased prevalence of vascular complications, for which new therapies targeting BPV remains a challenge. T2D, type 2 diabetes; eGFR, estimated glomerular filtration rate; BP, blood pressure; BPV, BP variability; RAS, renin-angiotensin system; ACEinh, angiotensin-converting-enzyme inhibitor; CCB, calcium channel blocker; CHD, coronary heart disease. A full color version of this figure is available at the Hypertension Research journal online.
1 Rothwell PM, Howard SC, Dolan E, O'Brien E, Dobson JE, Dahlöf B, Sever PS, Poulter NR. Prognostic significance of visit-to-visit variability, maximum systolic blood pressure, and episodic hypertension. Lancet 2010; 375: 895-905.

2 Nishimura M, Kato Y, Tanaka T, Todo R, Tone A, Yamada K, Ootani S, Kawabe $\mathrm{Y}$, Yoshizumi $\mathrm{H}$, Hoshiyama Y. Significance of estimating the glomerular filtration rate for the management of hypertension in type 2 diabetes with microalbuminuria. Hypertens Res 2013; 36: 705-710.

3 Muntner P, Shimbo D, Tonelli M, Reynolds K, Arnett DK, Oparil S. The relationship between visit-to-visit variability in systolic blood pressure and all-cause mortality in the general population: findings from NHANES III, 1988 to 1994. Hypertension 2011; 57: 160-166.

4 National Kidney Foundation. KDOQI Clinical Practice Guideline for Diabetes and CKD, 2012, update. Am J Kidney Dis 2012; 60: 850-886.

5 Weiner DE, Tighiouart $H$, Levey AS, Elsayed E, Griffith JL, Salem DN, Sarnak MJ. Lowest systolic blood pressure is associated with stroke in stages 3 to 4 chronic kidney disease. J Am Soc Nephrol 2007; 18: 960-966.

6 Rothwell PM, Howard SC, Dolan E, O'Brien E, Dobson JE, Dahlöf B, Poulter NR, Sever PS, ASCOT-BPLA, Trial MRC. InvestigatorsEffects of beta blockers and calcium-channel blockers on within-individual variability in blood pressure and risk of stroke. Lancet Neurol 2010; 9: 469-480. 
7 Dolan E, O'Brien E. Blood pressure variability: clarity for clinical practice. Hypertension 2010; 56: 179-181.

8 Eloot S, Schepers E, Barreto DV, Barreto FC, Liabeuf S, Van Biesen W, Verbeke F, Glorieux G, Choukroun G, Massy
Z, Vanholder R. Estimated glomerular filtration rate is a poor predictor of concentration for a broad range of uremic

toxins. Clin J Am Soc Nephrol 2011; 6: 1266-1273.

9 Mancia G, Grassi G, Giannattasio C, Seravalle G. Sympathetic activation in the pathogenesis of hypertension and progression of organ damage. Hypertension 1999; 34: 724-728.

10 Ksiazek A, Załuska W. Sympathetic overactivity in uremia. J Ren Nutr 2008; 18: 118-121. 